\title{
Expectations and Trust in Automated Vehicles
}

Qiaoning Zhang

University of Michigan

Ann Arbor, MI 48109, USA

qiaoning@umich.edu

X. Jessie Yang

University of Michigan

Ann Arbor, MI 48109, USA

xijyang@umich.edu

Lionel P. Robert Jr.

University of Michigan

Ann Arbor, MI 48109, USA

Iprobert@umich.edu

\begin{abstract}
A lack of trust is a major barrier to the adoptions of Automated Vehicles (AVs). Given the ties between expectation and trust, this study employs the expectation-confirmation theory to investigate in trust in AVs. An online survey was used to collect data including expectation, perceived performance, and trust in AVs from 443 participants which represent U.S. driver population. Using the polynomial regression and response surface methodology, we found that higher trust is engendered when perceived performance is higher than expectation, and perceived risk can moderate the relationship between expectation confirmation and trust in AVs. Results have important theoretical and practical implications.
\end{abstract}

\section{Author Keywords}

Automated Vehicles(AVs); Trust; Expectation Confirmation Theory; Performance; Risk.

\section{CCS Concepts}

-Human-centered computing $\rightarrow$ Human computer interaction (HCl); Haptic devices; User studies; Please use the 2012 Classifiers and see this link to embed them in the text: https://dl.acm.org/ccs/ccs_flat.cfm 


\section{Introduction}

A lack of trust may ultimately hinder the adoption of Automated Vehicles (AVs). Trust in AVs refers to the willingness of people to be vulnerable to the AVs' actions based on their expectations that the AVs is fully capable of driving even in conditions characterized by uncertainty and vulnerability [26, 31]. Trust is one of the primary drivers of AVs' adoption; therefore, understanding how to promote trust in AVs remains a vital challenge [7, 15, 29].

Expectations are likely to be important for understanding trust in AVs, yet this topic is vastly unexplored. Defined as beliefs about future actions involving the use of the technology associated with its capabilities, expectations are typically tied to consequences of current actions [3, 8]. People are more willing to use technology when it meets users' expectations [3]. Given the causal expectation-trust-adoption linkage, it becomes vital to examining this causal linkage in the specific case of AVs for both practice and research.

The Expectation-Confirmation Model (ECM) is developed to understand the impact of expectations on desirable outcomes. However, there is a gap in understanding trust using ECM in the AVs domain. Also, previous research highlight the importance of driving situations, which help define the potential risks associated with trust in AVs [11]. Nevertheless, it is not clear if and how driving situations can influence the relationship between expectation-confirmation and trust in AVs.

The goal of this study is to understand if and how expectationconfirmation can influence AV trust and how risk might alter this influence. An online survey study was conducted with 443 U.S. drivers. This study involved two types of weather conditions as the external environment factor: sunny and snowy; two kinds of AVs driving behaviors as the internal factor: normal drive and aggressive drive. The results found that highest trust was produced when perceived performance was higher than expected, and both internal and external factors had moderation effects on the relationship between expectation-confirmation and trust in AVs. The results of this study have crucial implications for AVs research and design.

\section{Background}

Trust in Automated Vehicles

Trust is important in the domain of automated vehicles (AVs). AVs, as the vehicles capable of driving autonomously without human supervision or intervention, have the potential to save lives, reduce crashes, congestion, fuel consumption, and pollution [1]. Despite many benefits provided by AVs, people still have trouble of accepting it. A lack of trust is a major barrier to their widespread adoption [2, 9 $14,21]$. Models and theories are used to understand how trust can influence people's behavioral intention of AVs [7, $15,29]$. The technology acceptance model (TAM) is used to show that trust strongly affects perceived usefulness, and both factors determine behavioral intention to adopt AVs [5]. In other words, trust is a major construct for predicting the AVs adoption.

Insights into factors that affect trust have drawn attention from researchers. A stream of literature investigates the factors influencing trust in AVs [7, 15, 29]. Improving driver's perception of the accuracy of the AVs, providing system information to help drivers predict and understand the operation of the AVs, and satisfying the driver's desire to know the driving situations are important for forming trustful relationship between drivers and AVs [5, 23].

In sum, AV trust is an important part of the research in the acceptance of AVs. There are many models and theories discussing factors affecting people's trust in automation and 
AVs. To enhance drivers' trust in and further acceptance of $\mathrm{AVs}$, more studies are needed to explore the factors that impact AV trust [9].

\section{Expectation-Confirmation Theory (ECT) and Trust}

The importance of expectations has significantly been emphasized with respects to attitudes toward and the adoption of technology. By definition, expectation can specifically be described as real-time representations of future situations, which involve the use of technology associated with its capabilities [3]. The initial expectation about a technology is formed based on existing knowledge and the interactions with communication channels or information source [40].

People's expectations may vary for the same technological product/service, depending on the quality and reliability of received information involving advertising, package information, media reports, media interviews and interpersonal communication [36]. In the AVs domain, prior research has found that expectations directly contribute to the acceptance and adoption of the AVs. With higher expectations, people would be more likely to accept and drive with AVs $[17,37]$. Expectation also has an indirect impact on the AVs adoptions. Positive expectations increase the generation of positive attitudes which are correlated with reliance on the $\mathrm{AVs}[20,30]$

Expectation-Confirmation Theory (ECT) and its counterpart Expectation-Disconfirmation Theory (EDT), both have been used to explain how technology adoption is influenced by whether someone's initial expectation of a technology is actually confirmed or dis-confirmed by their actual experience with that technology $[6,28,38]$. Described using the term "performance" in the EDT, experience illustrates an individual's post-usage belief about how the technology performed on the expectation attributes during the use period $[4,22]$. A confirmation occurs when performance meet expectation perfectly [12]. Disconfirmation includes positive disconfirmation when performance of the technology exceeds expectations, and negative disconfirmation when the technology performance fails to meet expectations [28, 34, 12].

Some previous literature states that trust can be linked to ECT because of the way the trust literature uses the terms "expect" and "expectation" in definition. For example, trust can be defined as expectations that an actor can be relied on, will be predictable, and will act fairly [39]. Trust is also described as "a set of expectations shared by all those involved in an exchange" [41]. Also, Trust theory has mentioned that trust grows when trust is positively confirmed but declines or is destroyed when trust is negatively disconfirmed [27]. These definitions and theories indicate that the progression of trust is related to the disconfirmation of trustrelated expectations and further relate trust to ECT [19, 24, $32,33]$. ECT is used to understand trust in technology. The results show that as disconfirmation becomes increasing positive, trust will increase. Negative disconfirmation impacts trust negatively. The results also show that negative disconfirmation will have a stronger negative effect on trust compared to the positive effect that positive disconfirmation will have [22]. Based on ECT, we argue that people generate a higher trust if their perceived $\mathrm{AVs}$ performance exceed the initial expectation.

H1: Positive disconfirmation lead to the highest trust in AVs.

The development of trust is related to the perception of risk because both attributes are related to uncertainty [11]. According to risk perception theory, risk-taking behavior is mediated by the level of perceived risk in the outcome of the behavior [25].In the context of driving, adverse weather is one of the critical causes of an elevated risk of traffic accidents and compromised traffic flow in the U.S. [18]. 
Also, internal characteristics which are the factors that occur inside the vehicle can also impact people's perceived risk of driving. Thus, one hypothesis is developed to better understand if and how the perceived risk introduced by weather and AVs driving behavior can influence the impact of expectation-confirmation on trust in AVs.

H2: Weather and AVs driving behavior will moderate the impact of expectation-confirmation on driver's trust in AVs.

\section{METHOD}

We conducted an online survey with 443 participants using a web-survey tool (Qualtric). This research complied with the American Psychological Association code of ethics and was approved by the University's Institutional Review Board. All participants provided informed consent.

\section{Participants and data collection}

A total of 443 U.S. drivers participated in this study. To create a representative sample, participants were screened for selected demographic characteristics that mark the driver population in the United States. A sampling profile was developed according to the demographic statistic from the U.S. Department of Transportation and AAA Foundation, which includes age, gender, race ethnicity, education, income, marital status, census region, frequency of driving, and driving experience [35]. Of the 443 participants, about $49 \%$ were male, and the average age was 47 years with a range from 18 to 84 years. Eighty-four percent of them drive almost every day, and others drive sometimes or rarely. Participants were compensated $\$ 5$ for completing the study.

Qualtrics Online Research Service was used to select a representative sample, U.S. drivers, and to conduct a webbased survey to test the hypotheses proposed above. We provided to Qualtrics the number of each subsample according to its demographic profile and sample size. The service selected and included the subjects in our participant poll if their demographic information were qualified for this study. Then, the central part of the online survey was distributed to collect data, in which each participant was required to watch four different videos and rated their perceptions after each of the videos.

\section{Independent variables}

This study is a $2 \times 2$ factorial design manipulated two independent variables related to factors that impact AVs externally (i.e. weather) and internally (i.e. AVs driving behavior). Figure 1-4 show the video screenshots for different conditions.

Weather conditions: As the influential external factor, weather conditions were manipulated by altering the weather in which the AV drives. Sunny weather conditions were coded as "1", snowy weather conditions were coded as " 2 ". When a participant watched the videos showing the sunny weather conditions, the road visibility and video brightness were much higher than the videos showing the snowy weather condition.

AVs driving behaviors: The driving behavior of $\mathrm{AVs}$ as the influential internal factor was measured as "1" if the car drove normally, and " 2 " if the car performed an aggressive driving behavior. Normal and aggressive driving behavior is differentiated by the number shown on the car speedometer and frequency of car shake. The videos showing aggressive driving behavior shaken more times, and the average speed was higher than the videos presenting normal driving behaviors.

AVs expectation: A three-item scale was appropriately worded to measure participants' AVs expectation before they watch the study videos. The questions based on a 7point Likert scale $(1=$ low, 7 = high $)$. 


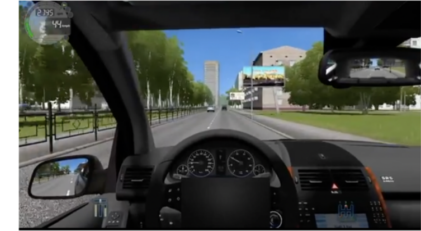

Figure 1: Normal AV driving in sunny weather

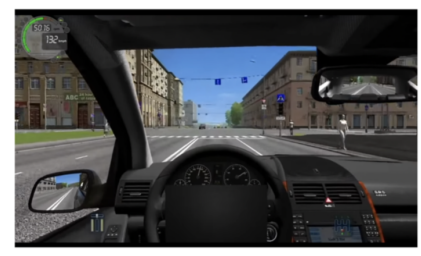

Figure 2: Aggressive AV driving in sunny weather

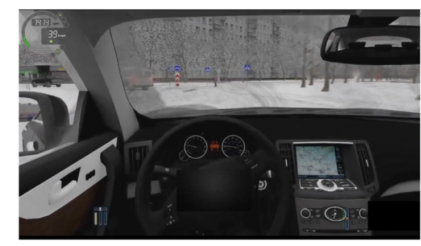

Figure 3: Normal AV driving in snowy weather

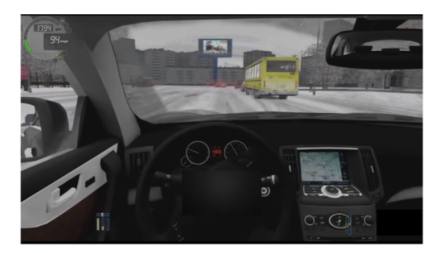

Figure 4: Aggressive AV driving in snowy weather
Perceived performance: Participants' perceived performance of AVs was measured with three items reflecting peoples' perception about what can realistically achieve or delivery by an AV. All the items were rated on seven-point Likert-type scales ( $1=$ low, 7 = high).

Dependent variable

Trust is the dependent variable in this study, which was measured using a questionnaire consisting of twelves sevenpoint Likert scales [16]. This scale is a highly validated instrument for gathering date about human and automated system trust. Questions in the trust questionnaire were appropriately revised to suit the AV context.

\section{Manipulation check of Risk Conditions}

We checked to confirm that the two different weather conditions and driving behaviors manipulated internal and external influential factors differently. The questions based on a 7-point Likert scale (1 = low, 7 = high) [10].

\section{RESULTS}

Preliminary Analysis

Construct internal consistency was supported by Cronbach's alpha with all construct reliability is at the acceptable threshold of .80 or above. To determine whether the measurement constructs were valid, we assessed convergent and discriminant validity through principal components analysis with varimax rotation that yielded a five-factor solution. All items loaded at .7 or above.

\section{Hypotheses Testing}

Polynomial regression analysis coupled with response surface methodology is used to test the hypotheses. $\mathrm{H} 1$ suggested that drivers have the highest trust in AVs if their perceived AV performance exceed their initial expectations. We
Table 1: Polynomial Regression Analysis Results

\begin{tabular}{|c|c|}
\hline & Regression coefficients \\
\hline Intercept/constant & $4.060^{\star *}$ \\
\hline Expectation(E) & $-0.069^{*}$ \\
\hline Perceived Performance (PP) & $0.451^{* *}$ \\
\hline$E^{2}$ & $-0.032^{*}$ \\
\hline$E * P P$ & $0.026^{*}$ \\
\hline$P P^{2}$ & 0.018 \\
\hline \multicolumn{2}{|c|}{${ }^{* *}$ Significant at the 0.001 level(2-tailed). } \\
*Significant at the 0.05 level(2-tailed).
\end{tabular}

propose:

$$
Z=b_{0}+b_{1} E+b_{2} P P+b_{3} E^{2}+b_{4} E P P+b_{5} P P^{2}+e(1)
$$

Where, $\mathrm{E}=$ Initial expectation, $\mathrm{PP}=$ Perceived Performance. Table 1 reports the polynomial regression results.

We compare the position of the ridge (First principle axis) to the line in the $X Y$ plane which contains all congruent predictor combinations: $X=Y$ (Congruence line) [13]. Based on the First principle equation: $\mathrm{Y}=p_{10}+p_{11} \mathrm{X}=10.422+4.091 \mathrm{X}$. The first principle axis shifted away from $X=Y$ as its intercept $p_{10}=10.422$ is significantly different from $0(\mathrm{p}<.001)$. Also, the slope of first principle axis $p_{11}=4.091$ is significantly different from 1 (the slope of $\mathrm{X}=\mathrm{Y}$ ) meaning the first principal axis is both shifted and rotated away from the $X=Y$. Thus, $\mathrm{H} 1$ was supported.

$\mathrm{H} 2$ suggests that $\mathrm{AVs}$ internal factor (AV driving behavior) and external factor (weather) will moderate the impact of (in)congruence between initial expectations and perceived performance on driver's trust in AVs. As shown in equation 5 , moderation effect can be tested by assessing the increment in $R^{2}$ yielded by the terms $X V, \mathrm{YV}, X^{2} V, X Y V$, and $Y^{2} V$. As putting AV driving behavior as the " $\mathrm{V}$ " into the 
model, the increment in $R^{2}$ is 4.127 ( $\left.\mathrm{p}<.05\right)$, the moderation effect of $A V s$ driving behavior thus supported. When treating weather as the " $\mathrm{V}$ " in the model, the increment in $R^{2}$ is $5.058(\mathrm{p}<.05)$ which indicates that weather has significant moderation effect as well. In sum, $\mathrm{H} 2$ was strongly supported.

\section{DISCUSSION}

This study sought to make contribution to the previous literature in the following ways. Firstly, this study contributes to $\mathrm{AVs}$ trust research by demonstrating the role of expectation on trust are related to perceived performance. This study is among the first to demonstrate that the role of expectations is relative to perceived performance. People's trust in AVs fluctuates with the relationship between expectations and performance. The impact of expectation on trust cannot be understood without considering perceived performance. Going forward, this means that future studies of AVs should consider examining the role of perceived performance along with expectations.

Secondly, this study provides evidences that not only are expectations important but confirming and disconfirming them are also important and have different impacts relative to perceived performance on AVs trust. The results of this study support the hypothesis of saying positively disconfirming expectations lead to the highest trust in AVs, This conclusion may seem intuitive but previous literature does not provide evidence about how much more benefit comes with exceeding compared to meeting expectations. This study shows that trust in AVs continuously increases as perceived performance increased to meet and exceed expectations, and the highest trust produced when the ratio between perceived performance and expectation becomes $4.091 / 1$
Finally, this study contributes to the literature by demonstrating that internal and external risk can moderate the effect of confirmation and disconfirmation on $\mathrm{AVs}$ trust as indicated in above contribution. This may because of the impact of risk perceptions on perceived performance. Uncertainty and potential loss introduced by a higher level of risk increase the possibility of individual's cognitive recognition about the product malfunctioning and usefulness which in turn impede the favorable evaluation.

\section{CONCLUSION}

This study extends our understandings in AVs trust by examining the role of expectation on trust in $\mathrm{AVs}$ as it related to perceived performance. Findings also indicate that the impact of expectation confirmation depends on internal and external risk factors including weather and driving behaviors respectively. With the polynomial regression model being supported, results of this study provide key insights into the role of expectation and perceived performance in trust in AV. This study opens up new topics and theoretical ground for future research to build and expand on the findings of this paper.

\section{REFERENCES}

[1] James M Anderson, Kalra Nidhi, Karlyn D Stanley, Paul Sorensen, Constantine Samaras, and Oluwatobi A Oluwatola. 2014. Autonomous vehicle technology: A guide for policymakers. Rand Corporation.

[2] Prateek Bansal, Kara M Kockelman, and Amit Singh. 2016. Assessing public opinions of and interest in new vehicle technologies: An Austin perspective. Transportation Research Part C: Emerging Technologies 67 (2016), 1-14. 
[3] Mads Borup, Nik Brown, Kornelia Konrad, and Harro Van Lente. 2006. The sociology of expectations in science and technology. Technology analysis \& strategic management 18, 3-4 (2006), 285-298.

[4] Ernest R Cadotte, Robert B Woodruff, and Roger L Jenkins. 1987. Expectations and norms in models of consumer satisfaction. Journal of marketing Research 24, 3 (1987), 305-314.

[5] Jong Kyu Choi and Yong Gu Ji. 2015. Investigating the importance of trust on adopting an autonomous vehicle. International Journal of Human-Computer Interaction 31, 10 (2015), 692-702.

[6] Her-Sen Doong and Hsiangchu Lai. 2008. Exploring usage continuance of e-negotiation systems: expectation and disconfirmation approach. Group Decision and Negotiation 17, 2 (2008), 111-126.

[7] Na Du, Jacob Haspiel, Qiaoning Zhang, Dawn Tilbury, Anuj K Pradhan, X Jessie Yang, and Lionel P Robert Jr. 2019. Look who's talking now: Implications of AV's explanations on driver's trust, AV preference, anxiety and mental workload. Transportation Research Part C: Emerging Technologies 104 (2019), 428-442.

[8] Norman T Feather. 1992. Values, valences, expectations, and actions. Journal of Social issues 48 , 2 (1992), 109-124.

[9] Jacob Haspiel, Na Du, Jill Meyerson, Lionel P Robert Jr, Dawn Tilbury, X Jessie Yang, and Anuj K Pradhan. 2018. Explanations and expectations: Trust building in automated vehicles. In Companion of the 2018 ACM/IEEE International Conference on Human-Robot Interaction. 119-120.

[10] Bob E Hayes, Jill Perander, Tara Smecko, and Jennifer Trask. 1998. Measuring perceptions of workplace safety: Development and validation of the work safety scale. Journal of Safety research 29, 3 (1998), 145-161.

[11] Kevin Anthony Hoff and Masooda Bashir. 2015. Trust in automation: Integrating empirical evidence on factors that influence trust. Human factors 57, 3 (2015), 407-434.

[12] Mohammad Alamgir Hossain and Mohammed Quaddus. 2012. Expectation-confirmation theory in information system research: A review and analysis. In Information systems theory. Springer, 441-469.

[13] Sarah Humberg, Steffen Nestler, and Mitja D Back. 2019. Response surface analysis in personality and social psychology: Checklist and clarifications for the case of congruence hypotheses. Social Psychological and Personality Science 10, 3 (2019), 409-419.

[14] Suresh Kumaar Jayaraman, Chandler Creech, Lionel P Robert Jr, Dawn M Tilbury, X Jessie Yang, Anuj K Pradhan, and Katherine M Tsui. 2018. Trust in AV: An uncertainty reduction model of AV-pedestrian interactions. In Companion of the 2018 ACM/IEEE International Conference on Human-Robot Interaction. 133-134.

[15] Suresh Kumaar Jayaraman, Chandler Creech, Dawn M Tilbury, Xi Jessie Yang, Anuj K Pradhan, Kate M Tsui, and Lionel Peter Robert. 2019.

Pedestrian Trust in Automated Vehicles: Role of Traffic Signal and AV Driving Behavior. Frontiers in Robotics and Al 6 (2019), 117.

[16] Jiun-Yin Jian, Ann M Bisantz, and Colin G Drury. 2000. Foundations for an empirically determined scale of trust in automated systems. International Journal of Cognitive Ergonomics 4, 1 (2000), 53-71. 
[17] Kanwaldeep Kaur and Giselle Rampersad. 2018. Trust in driverless cars: Investigating key factors influencing the adoption of driverless cars. Journal of Engineering and Technology Management 48 (2018), 87-96.

[18] Markku Kilpeläinen and Heikki Summala. 2007. Effects of weather and weather forecasts on driver behaviour. Transportation research part F: traffic psychology and behaviour 10, 4 (2007), 288-299.

[19] Euijin Kim and Suresh Tadisina. 2007. A model of customers' trust in e-businesses: micro-level inter-party trust formation. Journal of Computer Information Systems 48, 1 (2007), 88-104.

[20] Moritz Körber, Eva Baseler, and Klaus Bengler. 2018. Introduction matters: Manipulating trust in automation and reliance in automated driving. Applied ergonomics 66 (2018), 18-31.

[21] Miltos Kyriakidis, Riender Happee, and Joost CF de Winter. 2015. Public opinion on automated driving: Results of an international questionnaire among 5000 respondents. Transportation research part F: traffic psychology and behaviour 32 (2015), 127-140.

[22] Nancy K Lankton and Harrison D McKnight. 2012. Examining two expectation disconfirmation theory models: assimilation and asymmetry effects. Journal of the Association for Information Systems 13, 2 (2012), 1.

[23] Jiin Lee, Naeun Kim, Chaerin Imm, Beomjun Kim, Kyongsu Yi, and Jinwoo Kim. 2016. A question of trust: An ethnographic study of automated cars on real roads. In Proceedings of the 8th International Conference on Automotive User Interfaces and Interactive Vehicular Applications. ACM, 201-208.
[24] Roy J Lewicki, Barbara B Bunker, and others. 1996. Developing and maintaining trust in work relationships. Trust in organizations: Frontiers of theory and research 114 (1996), 139.

[25] Michael L Matthews and Andrew R Moran. 1986. Age differences in male drivers' perception of accident risk: The role of perceived driving ability. Accident Analysis \& Prevention 18, 4 (1986), 299-313.

[26] Roger C Mayer, James H Davis, and F David Schoorman. 1995. An integrative model of organizational trust. Academy of management review 20, 3 (1995), 709-734.

[27] Guido Möllering. 2001. The nature of trust: From Georg Simmel to a theory of expectation, interpretation and suspension. Sociology 35, 2 (2001), 403-420.

[28] Richard L Oliver. 1980. A cognitive model of the antecedents and consequences of satisfaction decisions. Journal of marketing research 17, 4 (1980), 460-469.

[29] L Petersen, L Robert, X Yang, and D Tilbury. 2019. Situational Awareness, Driver's Trust in Automated Driving Systems and Secondary Task Performance,". SAE International Journal of Connected and Automated Vehicles 2, 2 (2019).

[30] Yuna Ro and Youngwook Ha. 2019. A factor analysis of consumer expectations for autonomous cars. Journal of Computer Information Systems 59, 1 (2019), 52-60. 
[31] Lionel P Robert, Alan R Denis, and Yu-Ting Caisy Hung. 2009. Individual swift trust and

knowledge-based trust in face-to-face and virtual team members. Journal of Management Information Systems 26, 2 (2009), 241-279.

[32] Sandra L Robinson. 1996. Trust and breach of the psychological contract. Administrative science quarterly 41, 4 (1996).

[33] Denise M Rousseau, Sim B Sitkin, Ronald S Burt, and Colin Camerer. 1998. Not so different after all: A cross-discipline view of trust. Academy of management review 23, 3 (1998), 393-404.

[34] Richard A Spreng and Thomas J Page Jr. 2003. A test of alternative measures of disconfirmation. Decision Sciences 34, 1 (2003), 31-62.

[35] Tim Triplett, Rob Santos, Sandra Rosenbloom, and Brian Tefft. 2016. American driving survey: 2014-2015. (2016).

[36] David K Tse and Peter C Wilton. 1988. Models of consumer satisfaction formation: An extension. Journal of marketing research 25, 2 (1988), 204-212.
[37] lis P Tussyadiah, Florian J Zach, and Jianxi Wang. 2017. Attitudes toward autonomous on demand mobility system: The case of self-driving taxi. In Information and Communication Technologies in Tourism 2017. Springer, 755-766.

[38] Viswanath Venkatesh and Sandeep Goyal. 2010. Expectation disconfirmation and technology adoption: polynomial modeling and response surface analysis. MIS quarterly (2010), 281-303.

[39] Akbar Zaheer, Bill McEvily, and Vincenzo Perrone. 1998. Does trust matter? Exploring the effects of interorganizational and interpersonal trust on performance. Organization science 9, 2 (1998), 141-159.

[40] Valarie A Zeithaml, Anathanarayanan Parasuraman, Leonard L Berry, and Leonard L Berry. 1990. Delivering quality service: Balancing customer perceptions and expectations. Simon and Schuster.

[41] Lynne G Zucker. 1986. Production of trust: Institutional sources of economic structure, 1840-1920. Research in organizational behavior 8 (1986), 53-111. 